\title{
PLASMA EMISSION FROM HIGH VELOCITY IMPACTS OF MICROPARTICLES ONTO WATER ICE
}

\author{
R. TIMMERMANN and E. GRÜN \\ Max-Planck-Institut für Kernphysik \\ 6900 Heidelberg \\ Germany
}

\begin{abstract}
Collisions of icy objects play a major role in the outer solar system. The purpose of this investigation is the experimental study of plasma production by dust impacts on icy surfaces. Impact speeds ranged from 3 to $60 \mathrm{~km} / \mathrm{s}$. It was found that the dominant ion species which were released are both positive and negative water clusters. The impact charge yield from icy surfaces is approximately a factor 100 below that from previously studied gold surfaces.
\end{abstract}

\section{Introduction and Experimental Set-Up}

In the outer solar system water ice exists in abundance. Many satellites of the giant planets consist of ice. At these distances from the sun the lifetime of water ice particles against sublimation is long enough (Mukai, 1986), that even meteoroids may contain large fractions of ice. In a model by Goertz and Morfill (1983) the formation of spokes on Saturn's B ring is explained by impacts of meter sized meteoroids onto icy ring particles. Plasma is produced which expands in Saturn's magnetic field. In this plasma submicron particles can be charged and lift-off the ring by electrostatic forces overcoming the gravitational force, thus giving rise to the spoke phenomenon. The purpose of this study is the investigation of plasma production by impacts on icy surfaces.

In our experiments water ice targets were exposed to high speed dust particles and the produced plasma was analysed. Charges were collected by electrodes at bias of potentials 1000 to $3000 \mathrm{~V}$ which were positioned in front of the target. In one set of experiments the ions were collected after they had passed a $0.8 \mathrm{~m}$ long field-free drift tube. This way mass spectra of the ions were obtained. The target temperature ranged from 80 to $170 \mathrm{~K}$. Several hundred iron particles with masses ranging from $10^{-10}$ to $10^{-15} \mathrm{~g}$ were accelerated in the Heidelberg Van de Graaff to velocities ranging from 3 to $60 \mathrm{~km} / \mathrm{s}$, respectively. In addition, glass spheres in the mass range of $10^{-6}$ to $10^{-9} \mathrm{~g}$ were shot in the Munich plasma drag accelerator with speeds of up to $15 \mathrm{~km} / \mathrm{s}$.

A.C. Levasseur-Regourd and H. Hasegawa (eds.), Origin and Evolution of Interplanetary Dust, 375-378.

(C) 1991 Kluwer Academic Publishers, Printed in Japan. 

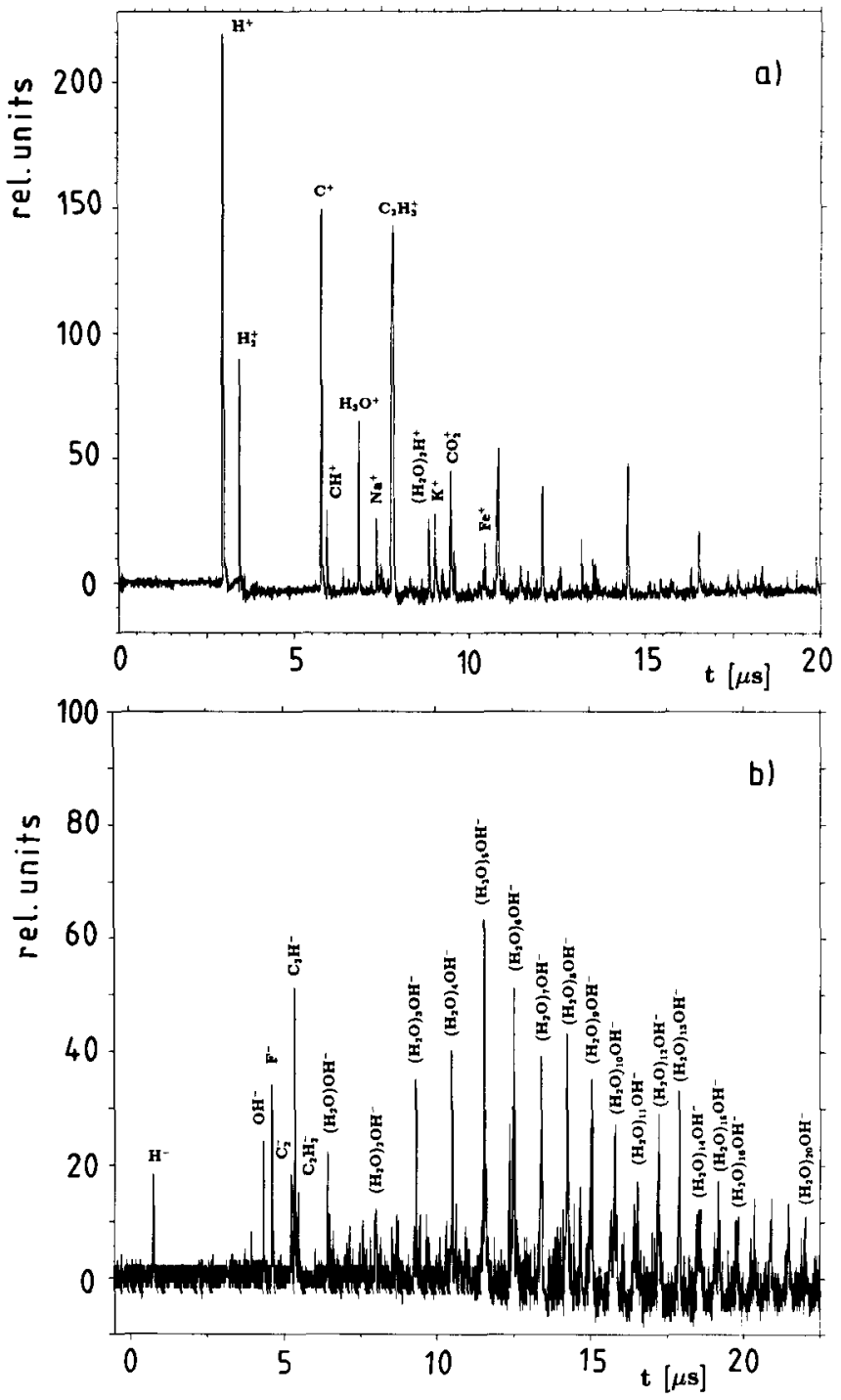

Figure 1. Time-of-flight mass spectra of ions released upon impacts of iron dust particles on ice.

a) Positive ion spectrum, impact speed $42.5 \mathrm{~km} / \mathrm{s}$.

b) Negative ion spectrum, impact speed $11.3 \mathrm{~km} / \mathrm{s}$.

\section{Experimental Results}

Positive and negative ions were mass analysed in a time-of-flight spectrometer. Figure 1 shows two examples of impact mass spectra obtained. 

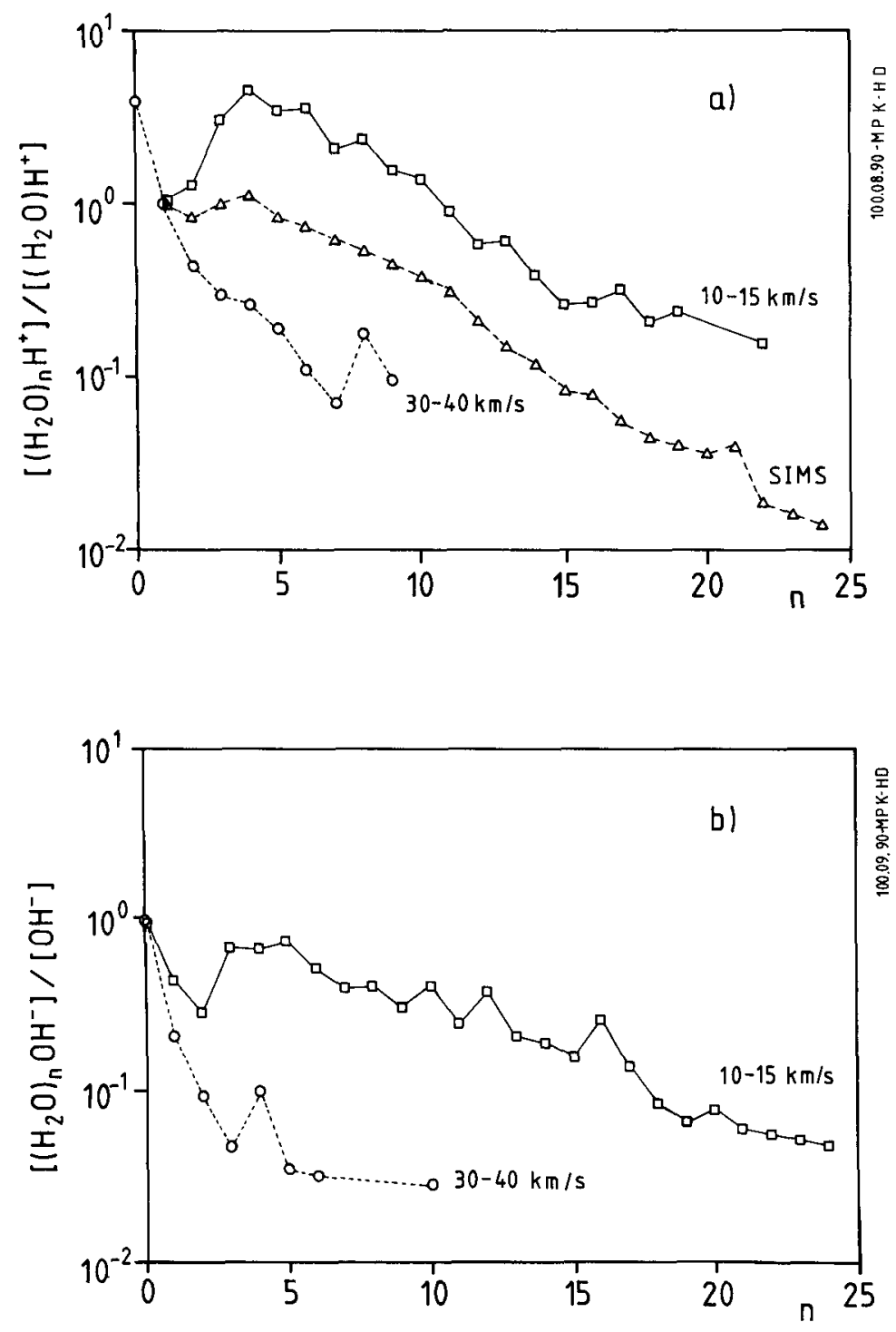

Figure 2. Cluster ion yield upon dust impacts on ice as a function of cluster size $n$ for two different impact speed intervals.
a) Positive ions, cluster ions produced by dust impacts and by SIMS (Estel et al., 1976) are compared
b) Negative ions.

At low velocities $(10-15 \mathrm{~km} / \mathrm{s})$ water clusters of the form $\left(\mathrm{H}_{2} \mathrm{O}\right)_{n} \mathrm{H}$ and $\left(\mathrm{H}_{2} \mathrm{O}\right){ }_{n} \mathrm{OH}$ could be detected where the number of water molecules $\mathrm{n}$ ranged up to 25 (cf. Figure 2). By increasing the velocity up to $40 \mathrm{~km} / \mathrm{s} \mathrm{n}$ decreased 
to small numbers; the ions released were composed mainly of $\mathrm{H}^{+}, \mathrm{H}_{3} \mathrm{O}^{+}, \mathrm{O}^{-}$ and $\mathrm{OH}^{-}$.

The total positive and negative charges released in these impact experiments fit to the empirical formula

$$
\mathrm{Q}^{+}=\mathrm{C} \mathrm{m}^{\alpha} \mathrm{v}^{\beta}
$$

with the following parameters $C=1.410^{-7}, \alpha=0.8$ and $\beta=2,45$ where $Q, m$ and $v$ is given in $C, g$ and $\mathrm{km} / \mathrm{s}$ and when the particle velocity is higher than $v=8 \mathrm{~km} / \mathrm{s}$. Below this velocity the yield for negative charges decreases to a value of a factor 0.2 compared to the emitted positive charges. In comparison to gold as target material (Griin, 1984) the amount of plasma released is roughly a factor of 100 lower in the case of an ice target. A temperature effect of the plasma yield was not found.

The experimentally determined values for the charge yield and the knowledge on the impact plasma composition will improve our understanding of the Saturnian ring system. The plasma yield for ice targets will set boundaries for the projectile sizes which cause spokes on Saturns B-ring. Sofar only estimates for the yields have been used (Morfill and Goertz, 1983).

Ip (1983) proposed that the inner edge of the B-ring is close to the distance at which ions generated at the ring plane will syphon off the ring and will be lost to the ionosphere. Impact plasma is one source for such ions. With realistic impact charge yields better life times of particles in that region of the rings are obtained.

ACKNOWLEDGEMENTS. The authors are indebted to $W$. Frisch and E. Igenbergs for their support to the experiments at the Munich dust accelerator. The work was supported by the Deutsche Forschungsgemeinschaft DFG.

\section{References}

Estel, J., Hoinkes, H., Kaarmann, H., Nahr, H. and Wilsch, H. (1976) On the problem of water adsorption on alkali halide cleavage planes, investigated by secondary ion mass spectroscopy, Surface Sci,, 54, 393-399

Goertz, C. and and Morfill, G. (1983) A model for the formation of spokes in Saturn's ring, Icarus, 53, 219-229

Griin, E. (1984) Impact ionization from gold, aluminum and PCB-Z, in Proc. of the Giotto Plasma Environment Working Group Meeting, ESA SP-224, 39-41

Ip, W.H. (1983) On plasma transport in the vicinity of the rings of Saturn: A siphon flow mechanism, J. Geophys. Res. 88, 819-822

Morfill, G.E. and Goertz C.K. (1983) Plasma clouds in Saturn's rings, Icarus, $55,111-123$

Mukai, T. (1986) Analysis of dirty water ice model for cometary dust, Astron. Astrophys., 164, 397-407 